\title{
Fall einer späten Diagnose des tumorartigen Stadiums eines Kaposi-Sarkoms
}

\author{
Case of a Late Diagnosis of a Tumorous Stage of Kaposi's Syndrome
}

Autoren

Institute

\section{A. Kuklin ${ }^{1}$, M. M. Kokhan' ${ }^{1}$, M. K. Kuklina²}

${ }^{1}$ Urales Wissenschaftliches Forschungsinstitut für Dermatovenerologie und Immunopathologie, Jekaterinburg, Russland

${ }^{2}$ Urale Föderale Boris Jelzin Universität, Jekaterinburg, Russland

\section{Bibliografie}

Dol http://dx.doi.org/

10.1055/s-0034-1377623

Online-Publikation: 20.8.2014

Akt Dermatol 2014; 40: 418-420

(c) Georg Thieme Verlag KG

Stuttgart · New York

ISSN 0340-2541

\section{Korrespondenzadresse}

Dr. med. Igor A. Kuklin

Urales Wissenschaftliches

Forschungsinstitut für

Dermatovenerologie und

Immunopathologie

Tscherbakov Str. 8

620076 Jekaterinburg

Russland

kuklin71@mail.ru

\section{Zusammenfassung}

$\nabla$

Der Artikel ist dem Fall einer tumorösen Systemerkrankung vaskulärer Genese aus der Zahl der Neubildungen der Dermis und des subkutanen Fettgewebes, dem Kaposi-Sarkom, gewidmet. Es werden die Fragen der Häufigkeit der Erkrankung, der Variabilität des klinischen Bildes und der Rechtzeitigkeit der Befunderstellung betrachtet. Es wird ein klinischer Fall einer späten Diagnose der Erkrankung beschrieben.

\section{Einleitung}

$\nabla$

Das Kaposi-Sarkom ist eine Tumorerkrankung vaskulärer Genese, bei der vorwiegend Haut, Lymphknoten und innere Organe befallen werden $[1,2]$. An der Erkrankung leiden Patienten im Alter von 50 bis 80 Jahren, Männer sind 9-15-mal öfter als Frauen betroffen. Ein Charakteristikum des Kaposi-Sarkoms sind Exantheme an den unteren Gliedmaßen - an der Rückseite der Füße und der Vorderseite der Unterschenkel, jedoch kann die gesamte Haut und Schleimhaut befallen werden: Augenlider, Nasenspitze, Schleimhaut des Mundes, Ohrmuscheln, Rücken, Bauch und Eichel $[3,4]$.

Das Kaposi-Sarkom ist eine polyätiologische Erkrankung. Derzeit sieht man als wichtige pathogenetische Faktoren die genetische Suszeptibilität zu dieser Erkrankung bei Personen mit Antigenen der Histokompatibilität HLA-DR5 sowie Virusinfektionen (HHV-8, CMV) und das HIV-Virus an. Es sind auch Fälle der Entwicklung eines Kaposi-Sarkoms bei Patienten unter immunsuppressiver Therapie nach Organtransplantation und bei Behandlung von Autoimmunerkrankungen [2,5-7] beschrieben.

Man unterscheidet 4 klinische Typen des KaposiSarkoms: einen klassischen (idiopathischen), einen endemischen (afrikanischen), einen immunsuppressiven und epidemischen (HIV-assozi- ierten) Typus. Klinisch manifestiert sich die Erkrankung in fleckigen, papulösen und tumorartigen Läsionen $[1,2,8]$.

Entsprechend dem Erkrankungsverlauf unterscheidet man: eine akute, eine subakute und eine chronische Form des Kaposi-Sarkoms. Für die akute Form sind frühe Generalisierung der Erkrankung, unentwegtes Fortschreiten, zahlreiche Läsionen der Haut und der Schleimhäute und Vergrößerung der Lymphknoten typisch. Ein letaler Ausgang nach einigen Monaten ist charakteristisch.

Bei der subakuten Form manifestiert sich die Erkrankung nicht so offensiv, ein letaler Ausgang kann 2-3 Jahre dauern.

Für die chronische Form des Kaposi-Sarkoms sind langsame Entwicklung und begrenzte Läsionen charakteristisch, erst im Verlauf kommt es zur Vergrößerung von Lymphknoten und einem Befall innerer Organe, die Lebensdauer der Patienten beträgt durchschnittlich $8-10$ Jahre $[3,9]$.

Histologisch zeigt sich ein angiogenetischer Spindelzelltumor mit charakteristischen eingeschlossenen Erythrozyten („Rolling“). Entzündlich können Lymphozyten, Plasmazellen und Makrophagen begleitend sein $[3,7,10]$.

Die Diagnose eines Kaposi-Sarkoms wird aufgrund des klinischen Bildes und der Ergebnisse der Histologie gestellt. Bestätigend kann eine HHV-8-Immunhistochemie gemacht werden.

Folgende Krankheiten sind u.a. zu differenzieren: Pseudo-Kaposi-Sarkom (Typ Mali), Pseudo-Kaposi-Sarkom (Typ Stewart-Bluefarb), Masson's Pseudo-Angiosarkom, bakterielle Angiomatose, Leiomyosarkom, Sarkoidose, Dermatofibrom, Hämangiom $[1,2,7,9]$.

Basispräparate in der Behandlung der Patienten mit Kaposi-Sarkom sind Zytostatika, Glukokortikoide, rekombinantes Interferon- $\alpha 1,-\alpha 2 b$ und $-\gamma$. Des Weiteren werden Strahlentherapie (Nahbestrahlung, Gamma-Distanztherapie) mit Gesamtdosen von 20-30 Gray, extrakorporale Fotopho- 


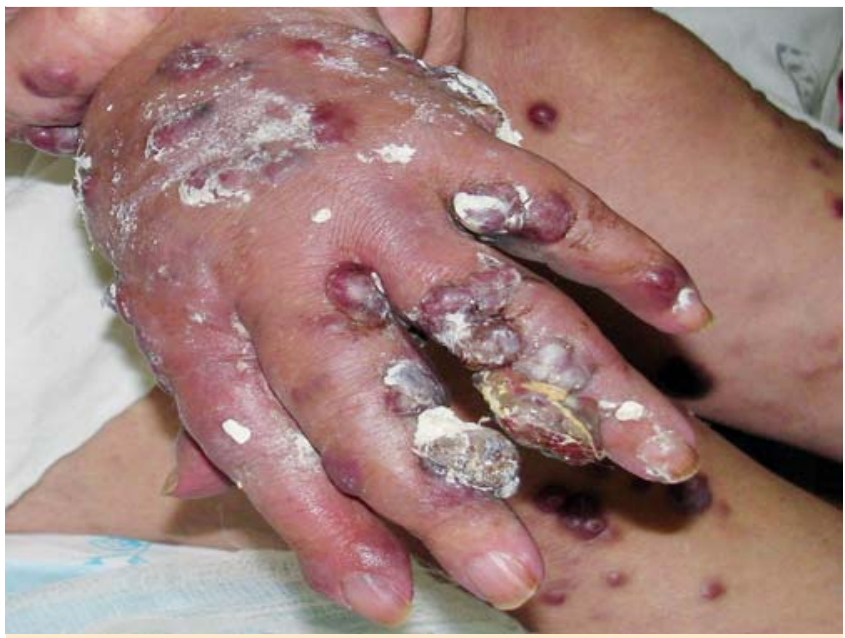

Abb. 1 Patientin, 74 Jahre alt, Diagnose: Kaposi-Sarkom, Tumorstadium. An der Haut der rechten Hand sind zahlreiche gruppierte Knoten mit einem Durchmesser von 1 bis zu $3 \mathrm{~cm}$.

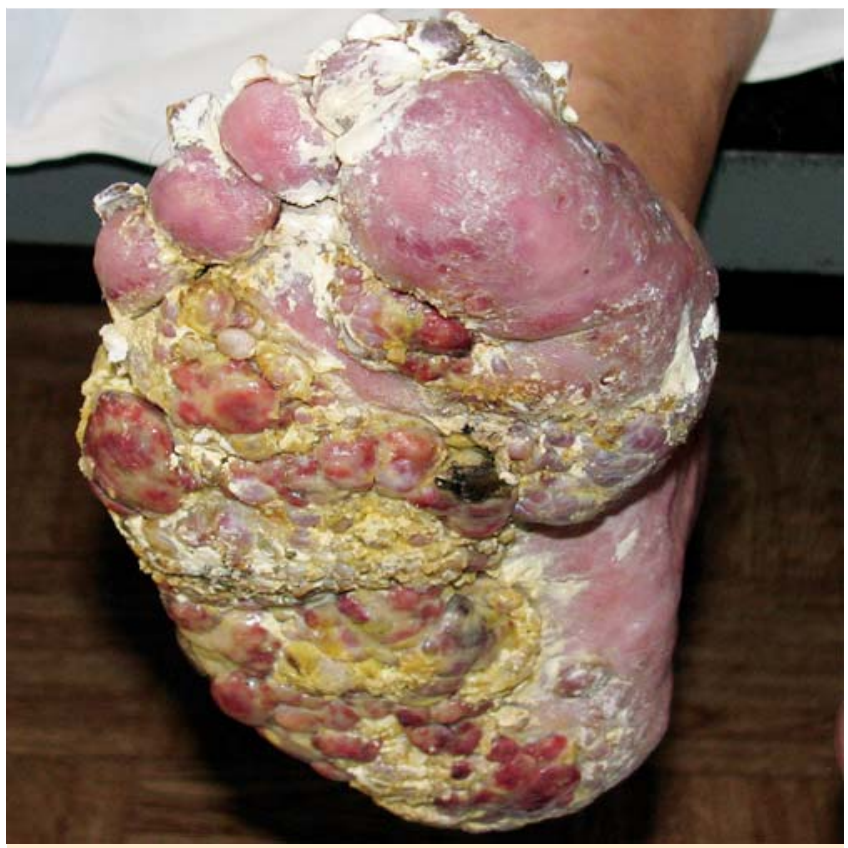

Abb.2 Das Ödem und die Vergrößerung des linken Fußes, die Knoten haben eine feste Konsistenz und einen Durchmesser von 3 bis zu $5 \mathrm{~cm}$.

rese und fotodynamische Therapie verwendet. Die Prognose der Erkrankung ist ungünstig $[1,2,6,11]$.

Als Beispiel für eine fortgeschrittene klinische Manifestation der Erkrankung präsentieren wir folgenden Fall.

\section{Kasuistik}

Die 74-jährige Patientin wandte sich mit Klagen über tumorähnliche Bildungen an der Haut der oberen und unteren Gliedmaßen, Ödeme der Unterschenkel, des Rumpfes und der Mundhöhle, eine allgemeine Schwäche, Schlafstörungen und einen Gewichtsverlust von $15 \mathrm{~kg}$ innerhalb der letzten 6 Monaten an die Klinik. Erstmals hatte die Patientin vor 8 Monaten an der Haut der Füße hellrote Flecken bemerkt, nach 2 Monaten entstanden dann ähnliche Elemente an der Haut der oberen und unteren Gliedmaßen, des Rumpfes und der Mundhöhle. Die Patientin be-

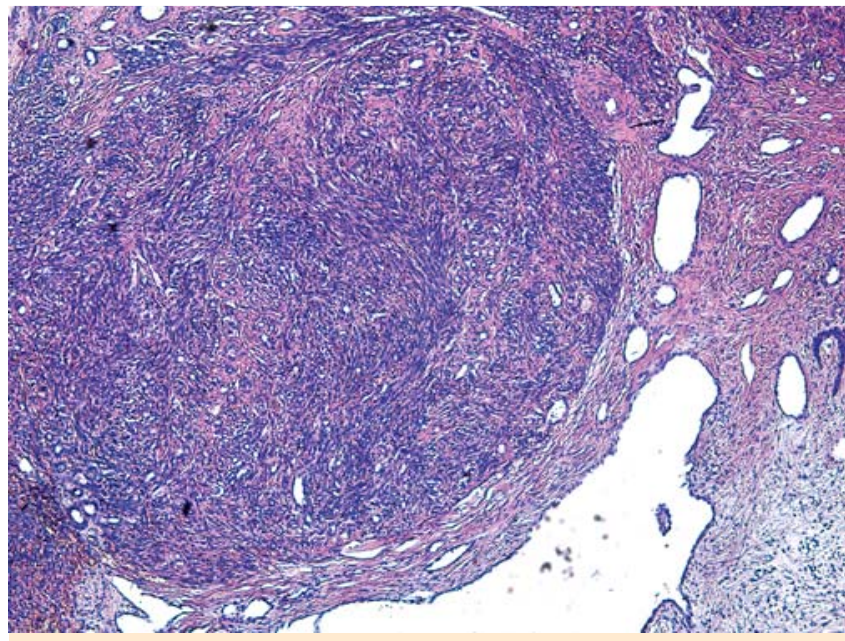

Abb.3 Relativ scharf begrenzte, nicht gekapselte Tumorknoten und dünnwandige Gefäßhöhlen (Färbung mit Hämatoxylin und Eosin, VergröBerung $\times 50$ ).

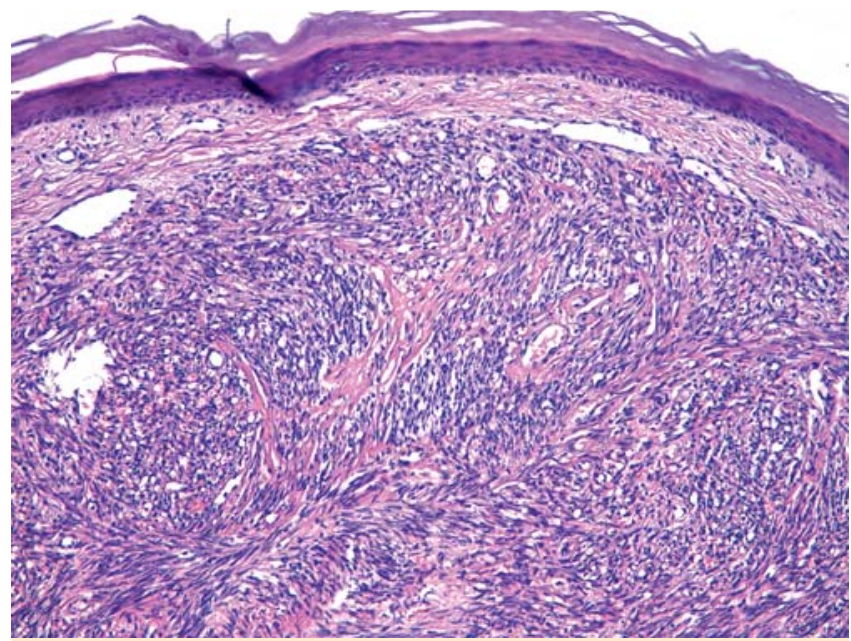

Abb.4 Aggregate von Spindelzellen mit Erythrozyten-gefüllten Spalträumen (Färbung mit Hämatoxylin und Eosin, Vergrößerung $\times 100$ ).

handelte sich bis dato selbst mit Aufgüssen und Absuden von Kräutern.

Die nicht betroffene Haut der Patientin hat eine physiologische Schattierung, normale Feuchtigkeit, der Turgor ist verringert. Sichtbare Schleimhäute sind rosa und feucht. Der Hautprozess hat einen symmetrisch verbreiteten Charakter, die Elemente befinden sich an der Haut des behaarten Kopfteils, des Gesichtes, des Rumpfes, der oberen und unteren Gliedmaßen. Zu sehen sind zahlreiche dichte, dunkelrote Knoten, die scharfe Grenzen, eine fest-elastische Konsistenz bei der Palpation und einen Durchmesser von 0,5 bis zu $3 \mathrm{~cm}$ haben. An der Haut der Hände und der Füße findet sich ein erosives Konglomerat der Knoten mit eitrigem Sekret und unangenehmen Geruch. Die Unterschenkel und Füße sind ödematös im Umfang vergrößert. Unter der Zunge sieht man einen roten Knoten mit einem Durchmesser bis $\mathrm{zu} 2 \mathrm{~cm}$, der schmerzlos bei der Palpation ist ( $\bullet$ Abb. 1, $\odot$ Abb.2). Labordaten: Blutbild: Senkung des Hämoglobins bis zu $78 \mathrm{~g} / \mathrm{dl}$, Beschleunigung der ESR bis zu $27 \mathrm{~mm} / \mathrm{h}$. Die allgemeine Urinuntersuchung: Eiweiß 0,048g/dl, Leukozyten 15 - 18 im Blickfeld. Die biochemische Blutanalyse: Erhöhung der alkalischen Phosphatase bis zu 212U/l. Die Blutuntersuchungen auf RW, HbsAg und HIV sind negativ. 
Histologische Untersuchung: In der Dermis sind deutliche Tumorknoten aus den sich verschlingenden Bündeln der Spindelzellen mit geringgradigem nuklearem Polymorphismus zu sehen, die durch Spalträume mit Erythrozyten geteilt sind. In der Peripherie der Knoten finden sich dünnwandige Gefäßlakunen nach Art eines kavernösen Hämangioms sowie lymphohistiozytäres Infiltrat. Somit bestätigte sich ein Kaposi-Sarkom im Tumorstadium (৫ Abb. 3, $\odot$ Abb.4).

Angesichts des Tumorstadiums des Kaposi-Sarkoms wurde die Patientin in das regionale onkologische Zentrum zur spezifischen Therapie überwiesen.

\section{Interessenkonflikt}

$\nabla$

Die Autoren geben an, dass kein Interessenkonflikt besteht.

\section{Abstract}

\section{Case of a Late Diagnosis of a Tumorous Stage of Kaposi's Syndrome \\ $\nabla$}

The article provides information on frequency, prevalence, and clinical manifestations of Kaposi's sarcoma and shows a case of a late clinical diagnosis of the disease.

\section{Literatur}

1 Kubanowa AA. Klinische Empfehlungen. Dermatovenerologie 2010: $214-228$

2 Jelkin WD, Mitrjukowskij LS, Sedowa TG. Ausgewählte Dermatologie. Seltene Dermatosen und dermatologische Syndrome. Das illustrierte Nachschlagewerk für die Diagnostik und Behandlung der Dematosen. Perm 2004: 570 - 572

3 Bernardini B, Faggion D, Calabró L et al. Imiquimod Treatment of Classical Kaposi's Sarcoma. Acta Derm Venereol 2010; 90: 417-418

4 Molotschkow AW, Kartaschowa MG, Prokofjew AA. Fall der erfolgreichen Anwendung der fotodynamischen Therapie in der Behandlung des Kaposi-Sarkoms auf dem männlichen Glied. Boten der Dermatologie und der Venerologie 2010; 5: 92 - 95

5 Nowikowa NW, Tschistjakowa IA, Resajkina AW. Kaposi-Sarkom: moderne Daten über die Pathogenese, Klinik und Therapie. Boten der Dermatologie und der Venerologie 2002; 3: 44-49

6 Kadyrowa EL, Kartaschowa MG, Molotschkow AW. Manifestation des Humanen Herpesvirus Typ 8 in Gewebe des Urogenitaltrakts bei idiopathischem Sarkom AW. Russische Zeitschrift der Haut- und Geschlechtskrankheiten 2001; 6: 7-10

7 Rukscha TG, Aksenenko MB, Chorschewskij WA et al. Fall des KaposiSarkoms: die Anwendung der immunhistochemischen Forschung für die Identifikation des Humanen Herpesvirus Typ 8. Boten der Dermatologie und der Venerologie 2013; 5: 115-118

8 Galil-Ogly GA, Molotschkow WA, Sergejew JW. Dermato-Oncologie. Verlag: Medizin für alle; 2005: 872

9 Wolff K, Johnson RA, Saavedra AP et al. Fitzpatricks Color Atlas \& Synopsis of Clinical Dermatology. New York: McGrawHill; 2007: 629-634

10 Molotschkow AW, Tschernowa WA, Watasin AW et al. Positive serologische Reaktionen zu HHV-8 - der Risikofaktor der Entwicklung des Kaposi-Sarkoms bei den Nierenempfängern. Russische Zeitschrift der Haut- und Geschlechtskrankheiten 2002; 6: 4-7

11 Muchina EW, Lamotkin IA, Kapustina OG. Behandlung des idiopathischen Kaposi-Sarkoms. Ambulatorische Chirurgie 2008; 2: 33-37 